\title{
EXISTENCE CRITERIA AND CLASSIFICATION SCHEMES FOR POSITIVE SOLUTIONS OF SECOND-ORDER NONLINEAR DIFFERENCE SYSTEMS
}

WAN-TONG LI, CAN-YUN HUANG, AND SUI SUN CHENG

Received 1 May 2006; Accepted 19 July 2006

Classification schemes for positive solutions of a class of second-order nonlinear difference systems are given in terms of their asymptotic magnitudes; and necessary as well as sufficient conditions for the existence of these solutions are also provided. Finally, some examples are given to illustrate the main results.

Copyright (c) 2006 Wan-Tong Li et al. This is an open access article distributed under the Creative Commons Attribution License, which permits unrestricted use, distribution, and reproduction in any medium, provided the original work is properly cited.

\section{Introduction}

The asymptotic behavior of solutions of nonlinear difference equations is of particular interest in iterative computational schemes and discrete time dynamic models. Therefore, it is the subject of many investigations $[1,2,4,5,8-14,18,20]$.

In this paper, we are concerned with a class of two-dimensional second-order nonlinear difference systems of the form

$$
\begin{gathered}
\Delta^{2} x_{n}=a_{n} f\left(y_{n}\right), \\
\Delta^{2} y_{n}=-b_{n} g\left(x_{n}\right),
\end{gathered}
$$

where $\left\{a_{n}\right\}_{n=n_{0}}^{\infty}$ and $\left\{b_{n}\right\}_{n=n_{0}}^{\infty}$ are real, nontrivial sequences such that $a_{n} \geq 0$ and $b_{n} \geq 0$ for $n \geq n_{0}, f$ and $g$ are continuous real-valued and increasing functions on the real line $\mathbb{R}$ and satisfy $x f(x)>0, x g(x)>0$ for $x \neq 0$.

Existence and uniqueness theorem for solutions of (1.1) is easily established. Indeed, given $x_{0}, x_{1}, y_{0}$ and $y_{1}$, we can calculate

$$
x_{2}=2 x_{1}-x_{0}+a_{0} f\left(y_{0}\right), \quad y_{2}=2 y_{1}-y_{0}-b_{0} g\left(x_{0}\right), \ldots
$$

successively in a unique manner. The corresponding sequence $\left\{\left(x_{n}, y_{n}\right)\right\}_{n=n_{0}}^{\infty}$ will be called a solution of (1.1). 
A solution $\left\{\left(x_{n}, y_{n}\right)\right\}$ of $(1.1)$ is said to be positive if both $\left\{x_{n}\right\}$ and $\left\{y_{n}\right\}$ are positive. Positive solutions of (1.1) are interesting for many reasons. For instance, when $a_{n} \equiv 1$ and $f(u)=u$, we see from $(1.1)$ that

$$
\Delta^{2} y_{n}=\Delta^{4} x_{n}=-b_{n} g\left(x_{n}\right)
$$

Therefore, a positive solution of (1.1) yields a positive and strictly concave solution of the fourth-order nonlinear difference equation

$$
\Delta^{4} x_{n}+b_{n} g\left(x_{n}\right)=0
$$

Other difference equations such as

$$
\begin{gathered}
\Delta^{4} x_{n-1}+p_{n} f\left(x_{n}\right)=0, \quad \Delta^{4} x_{n-1}+p_{n}\left|x_{n}\right|^{\gamma} \operatorname{sign} x_{n}=0, \\
\Delta^{2}\left(r_{n-1} \Delta^{2} x_{n-1}\right)+p_{n} f\left(x_{n}\right)=0
\end{gathered}
$$

can also be written in the form (1.1), which have been explored to some extent in a number of studies $[3,6,7,15-17,19]$. We will be concerned with existence criteria as well as classification schemes for positive solutions of (1.1).

We remark that our system (1.1) is a discrete analog of a second-order differential system of the form

$$
x^{\prime \prime}=a(t) f(y), \quad y^{\prime \prime}=-b(t) g(x),
$$

which can be interpreted as the governing equations of the motion of a particle moving in a plane under a nonautonomous plane force field. Therefore the study of (1.1) will also lead to useful complementary information for the differential systems. On the other hand, (1.1) can also be written as a first-order difference system. For results related to these systems, the reader can refer to [7]. We remark, however, that our approach here is more natural and avoids systems with four equations.

Our system (1.1) is naturally classified into four classes: (i) $\sum_{s=n_{0}}^{\infty} a_{s}=\infty$ and $\sum_{s=n_{0}}^{\infty} b_{s}=$ $\infty$; (ii) $\sum_{s=n_{0}}^{\infty} a_{s}=\infty$ and $\sum_{s=n_{0}}^{\infty} b_{s}<\infty$; (iii) $\sum_{s=n_{0}}^{\infty} a_{s}<\infty$ and $\sum_{s=n_{0}}^{\infty} b_{s}=\infty$; and (iv) $\sum_{s=n_{0}}^{\infty} a_{s}<\infty$ and $\sum_{s=n_{0}}^{\infty} b_{s}<\infty$.

For this reason, we will employ the following notations:

$$
A_{n}=\sum_{s=n}^{\infty} a_{s}, \quad B_{n}=\sum_{s=n}^{\infty} b_{s}, \quad n \geq n_{0} .
$$

In the following section, we will discuss the case $A_{n_{0}}=\infty$ and $B_{n_{0}}=\infty$. The cases where $A_{n_{0}}=\infty$ and $B_{n_{0}}<\infty, A_{n_{0}}<\infty$ and $B_{n_{0}}<\infty$, and $A_{n_{0}}<\infty$ and $B_{n_{0}}=\infty$ will be studied in Sections 3, 4, and 5, respectively. In Section 6, we give some examples to illustrate our results.

2. The case $A_{n_{0}}=\infty$ and $B_{n_{0}}=\infty$

In this section, we always assume that $A_{n_{0}}=\infty$ and $B_{n_{0}}=\infty$. We assert that there exist no positive solutions of (1.1). 
Theorem 2.1. Suppose that $A_{n_{0}}=\infty$ and $B_{n_{0}}=\infty$. Then there exist no positive solutions of (1.1).

Proof. Suppose that $\left\{\left(x_{n}, y_{n}\right)\right\}$ is a solution of (1.1) such that $x_{n}>0$ and $y_{n}>0$ for $n \geq n_{0}$. Then, from (1.1) we have $\Delta^{2} y_{n}<0$ for $n \geq n_{0}$, which implies that $\left\{\Delta y_{n}\right\}$ is decreasing. Therefore, there are two possibilities: (i) $\Delta y_{n}>0$ for $n \geq n_{0}$ and (ii) $\Delta y_{n}<0$ for $n \geq n_{0}$.

If $\Delta y_{n}>0$ for $n \geq n_{0}$, then $\left\{y_{n}\right\}$ is an increasing sequence. Since $y_{n}>0$ for $n \geq n_{0}$, then $y_{n} \geq y_{n_{0}}>0$ for $n \geq n_{0}$. From the first equation of (1.1) we have $\Delta^{2} x_{n}>0$ for $n \geq n_{0}$ and hence

$$
\Delta x_{n}=\Delta x_{n_{0}}+\sum_{k=n_{0}}^{n-1} a_{k} f\left(y_{k}\right) \geq \Delta x_{n_{0}}+f\left(y_{n_{0}}\right) \sum_{k=n_{0}}^{n-1}, \quad a_{k} \longrightarrow \infty
$$

as $n \rightarrow \infty$, which implies that there exists an integer $n_{1} \geq n_{0}$ such that $x_{n} \geq x_{n_{1}}>0$ for $n \geq n_{1}$. From the second equation of (1.1), we have

$$
\Delta y_{n}=\Delta y_{n_{1}}-\sum_{k=n_{1}}^{n-1} b_{k} g\left(x_{k}\right) \leq \Delta y_{n_{1}}-g\left(x_{n_{1}}\right) \sum_{k=n_{1}}^{n-1}, \quad b_{k} \longrightarrow-\infty
$$

as $n \rightarrow \infty$, which contradicts the fact that $\Delta y_{n}>0$ for $n \geq n_{0}$.

If $\Delta y_{n}<0$ for $n \geq n_{0}$, then from $\Delta^{2} y_{n}<0$ for $n \geq n_{0}$, it follows that $\left\{\Delta y_{n}\right\}$ is decreasing, and hence there exists a constant $c>0$ such that $\Delta y_{n} \leq-c$ for $n \geq n_{2} \geq n_{0}$, which means that $y_{n} \leq y_{n_{2}}-\sum_{k=n_{2}}^{n-1} c \rightarrow-\infty$ as $n \rightarrow \infty$, and so there exists an integer $n_{3} \geq n_{2}$ such that $y_{n}<0$ for $n \geq n_{3}$. This is a contradiction and completes the proof.

\section{The case $A_{n_{0}}=\infty$ and $B_{n_{0}}<\infty$}

Assume that $A_{n_{0}}=\infty$ and $B_{n_{0}}<\infty$. If $\left\{\left(x_{n}, y_{n}\right)\right\}_{n=n_{0}}^{\infty}$ is a positive solution of (1.1), that is to say, $x_{n}>0$ and $y_{n}>0$ for $n \geq n_{0}$, then, in view of (1.1), we have $\Delta^{2} x_{n}>0$ and $\Delta^{2} y_{n}<0$ for $n \geq n_{0}$, which imply that $\left\{\Delta x_{n}\right\}$ is increasing and $\left\{\Delta y_{n}\right\}$ is decreasing. Hence $\left\{x_{n}\right\}$ and $\left\{y_{n}\right\}$ are monotonic sequences. By the second equation of (1.1), we have

$$
\Delta y_{n}=\Delta y_{n_{0}}-\sum_{k=n_{0}}^{n-1} b_{k} g\left(x_{k}\right), \quad n \geq n_{0} .
$$

If there exists an integer $n_{1} \geq n_{0}$ such that $\Delta y_{n}<\Delta y_{n_{1}}<0$ for $n \geq n_{1}$, then

$$
y_{n}=y_{n_{1}}+\sum_{k=n_{1}}^{n-1} \Delta y_{k} \leq y_{n_{1}}+\sum_{k=n_{1}}^{n-1}, \quad \Delta y_{n_{1}} \longrightarrow-\infty
$$

as $n \rightarrow \infty$, which contradicts the assumption $y_{n}>0$ for $n \geq n_{0}$. Hence $\Delta y_{n}>0$ for $n \geq n_{0}$ and $\lim _{n \rightarrow \infty} \Delta y_{n}=c \geq 0$, which implies that $\lim _{n \rightarrow \infty} y_{n}=\infty$ or $\lim _{n \rightarrow \infty} y_{n}=\beta>0$.

By the first equation of (1.1), we have

$$
\Delta x_{n}=\Delta x_{n_{0}}+\sum_{k=n_{0}}^{n-1} a_{k} f\left(y_{k}\right) \geq \Delta x_{n_{0}}+f\left(y_{n_{0}}\right) \sum_{k=n_{0}}^{n-1}, \quad a_{k} \longrightarrow \infty
$$

as $n \rightarrow \infty$, and so, $\lim _{n \rightarrow \infty} x_{n}=\infty$. 


\section{Positive solutions of nonlinear difference systems}

In view of the above discussions, we may now make the following classification. Let $C$ be the set of all positive solutions of (1.1). Then we have the following result.

Theorem 3.1. Suppose that $A_{n_{0}}=\infty$ and $B_{n_{0}}<\infty$. Then any positive solutions of (1.1) must belong to the following classes:

$$
\begin{aligned}
& C(\infty, \alpha)=\left\{\left(x_{n}, y_{n}\right) \in C \mid \lim _{n \rightarrow \infty} x_{n}=\infty, \lim _{n \rightarrow \infty} y_{n}=\beta>0\right\}, \\
& C(\infty, \infty)=\left\{\left(x_{n}, y_{n}\right) \in C \mid \lim _{n \rightarrow \infty} x_{n}=\infty, \lim _{n \rightarrow \infty} y_{n}=\infty\right\} .
\end{aligned}
$$

In order to further justify our classification schemes, we derive several sufficient conditions for the existence of each type of positive solutions.

Theorem 3.2. Suppose that $A_{n_{0}}=\infty$ and $B_{n_{0}}<\infty$. A sufficient condition for (1.1) to have a positive solution $\left\{\left(x_{n}, y_{n}\right)\right\}$ which belongs to $C(\infty, \alpha)$ is that

$$
\sum_{k=n_{0}}^{\infty} \sum_{s=k}^{\infty} b_{s} g\left(\sum_{r=n_{0}}^{s-1} \sum_{t=n_{0}}^{r-1} a_{t} f(c)\right)<\infty
$$

for some $c>0$ and

$$
\sum_{k=n_{0}}^{\infty} \sum_{s=n_{0}}^{k-1} a_{s} f(d)=\infty
$$

for any $d>0$.

Proof. Choose $N$ so large that

$$
\sum_{k=N}^{\infty} \sum_{s=k}^{\infty} b_{s} g\left(\sum_{r=N}^{s-1}\left(\sum_{t=N}^{r-1} a_{t} f(c)\right)\right)<\frac{c}{2} .
$$

Let $X$ be the set of all bounded real-valued sequences $\left\{y_{n}\right\}$ with norm $\sup _{n \geq N}\left|y_{n}\right|$. Then $X$ is a Banach space. We define a subset $\Omega$ of $X$ as follows:

$$
\Omega=\left\{y_{n} \in X \mid \frac{c}{2} \leq y_{n} \leq c, n \geq N\right\}
$$

Then $\Omega$ is bounded, convex and closed subset of $X$. Let us further define an operator $F: \Omega \rightarrow X$ as follows:

$$
(F y)_{n}=c-\sum_{k=n}^{\infty} \sum_{s=k}^{\infty} b_{s} g\left(\sum_{r=N}^{s-1} \sum_{t=N}^{r-1} a_{t} f\left(y_{t}\right)\right), \quad n \geq N .
$$

The mapping $F$ has the following properties. First of all, $F$ maps $\Omega$ into $\Omega$. Indeed, 
if $y \in \Omega$, then

$$
\begin{aligned}
c & \geq(F y)_{n}=c-\sum_{k=n}^{\infty} \sum_{s=k}^{\infty} b_{s} g\left(\sum_{r=N}^{s-1} \sum_{t=N}^{r-1} a_{t} f\left(y_{t}\right)\right) \\
& \geq c-\sum_{k=N}^{\infty} \sum_{s=k}^{\infty} b_{s} g\left(\sum_{r=N}^{s-1} \sum_{t=N}^{r-1} a_{t} f(c)\right) \geq \frac{c}{2} .
\end{aligned}
$$

Next, we show that $F$ is continuous. Let $y^{(l)} \in \Omega$ such that $\lim _{l \rightarrow \infty}\left\|y^{(l)}-y\right\|=0$. Since $\Omega$ is closed, $y \in \Omega$. Then by (3.9), we have

$$
\begin{aligned}
\left|\left(F y^{(l)}\right)_{n}-(F y)_{n}\right| & =\left|\sum_{k=n}^{\infty} \sum_{s=k}^{\infty} b_{s} g\left(\sum_{r=N}^{s-1} \sum_{t=N}^{r-1} a_{t} f\left(y_{t}^{(l)}\right)\right)-\sum_{k=n}^{\infty} \sum_{s=k}^{\infty} b_{s} g\left(\sum_{r=N}^{s-1} \sum_{t=N}^{r-1} a_{t} f\left(y_{t}\right)\right)\right| \\
& \leq \sum_{k=n}^{\infty} \sum_{s=k}^{\infty} b_{s}\left|g\left(\sum_{r=N}^{s-1} \sum_{t=N}^{r-1} a_{t} f\left(y_{t}^{(l)}\right)\right)-g\left(\sum_{r=N}^{s-1} \sum_{t=N}^{r-1} a_{t} f\left(y_{t}\right)\right)\right| .
\end{aligned}
$$

By the continuity of $f$ and $g$ and Lebesgue's dominated convergence theorem, it follows that

$$
\limsup _{l \rightarrow \infty}\left|\left(F y^{(l)}\right)_{n}-(F y)_{n}\right|=0
$$

This shows that $\lim _{l \rightarrow \infty}\left\|F y^{(l)}-F y\right\|=0$, that is, $F$ is continuous.

Finally, we will show that $F \Omega$ is precompact. Let $y \in \Omega$ and $m, n \geq N$. Then, we have, for $m>n$,

$$
\left|(F y)_{m}-(F y)_{n}\right| \leq \sum_{s=n}^{m-1} \sum_{s=k}^{\infty} b_{s} g\left(\sum_{r=N}^{s-1} \sum_{t=N}^{r-1} a_{t} f\left(y_{t}\right)\right) \leq \sum_{s=n}^{\infty} \sum_{s=k}^{\infty} b_{s} g\left(\sum_{r=N}^{s-1} \sum_{t=N}^{r-1} a_{t} f\left(y_{t}\right)\right) .
$$

In view of (3.5), this means that $F \Omega$ is precompact.

By Schauder's fixed point theorem, we conclude that there exists a $y \in \Omega$ such that $y=F y$. Set $x_{n}=\sum_{r=N}^{n-1} \sum_{t=N}^{r-1} a_{t} f\left(y_{t}\right)$. Then

$$
x_{n} \geq \sum_{r=N}^{n-1} \sum_{t=N}^{r-1} a_{t} f\left(\frac{c}{2}\right) \longrightarrow \infty \quad \text { as } n \rightarrow \infty,
$$

and hence $\lim _{n \rightarrow \infty} x_{n}=\infty$. On the other hand,

$$
y_{n}=(F y)_{n}=c-\sum_{k=n}^{\infty} \sum_{s=k}^{\infty} b_{s} g\left(x_{s}\right)
$$

which implies that $\lim _{n \rightarrow \infty} y_{n}=c$. The proof is complete. 
6 Positive solutions of nonlinear difference systems

Theorem 3.3. Suppose that $A_{n_{0}}=\infty$ and $B_{n_{0}}<\infty$. A sufficient condition for (1.1) to have a positive solution $\left\{\left(x_{n}, y_{n}\right)\right\}$ which belongs to $C(\infty, \infty)$ is that

$$
\sum_{k=n_{0}}^{\infty} a_{k} f(c k)<\infty
$$

for some $c>0$ and

$$
\sum_{k=n_{0}}^{\infty} b_{k} g(d k)<\infty
$$

for some $d>0$.

Proof. Suppose that (3.16) and (3.17) hold. Then there exists $N \geq n_{0}$ such that

$$
\sum_{k=N}^{\infty} a_{k} f(2 c k)<d, \quad \sum_{k=N}^{\infty} k g(2 d k)<c .
$$

Let $X$ be the Banach space of all real-valued sequences $\left\{\left(x_{n}, y_{n}\right)\right\}$ endowed with the norm

$$
\|(x, y)\|=\max \left\{\sup _{n \geq N}\left|\frac{x_{n}}{n}\right|, \sup _{n \geq N}\left|\frac{y_{n}}{n}\right|\right\}
$$

and with the usual pointwise ordering $\leq$. Define a subset $\Omega$ of $X$ as follows:

$$
\Omega=\left\{(x, y) \in X \mid d n \leq x_{n} \leq 2 d n, c n \leq y_{n} \leq 2 c n, n \geq N\right\} .
$$

For any subset $B$ of $\Omega$, it is obvious that $\inf B \in \Omega$ and $\sup B \in \Omega$. Let us further define an operator $F: \Omega \rightarrow X$ as follows: for $(x, y) \in X, F(x, y)=(u, v)$ and

$$
u_{n}=d n+\sum_{k=N}^{n-1} \sum_{s=N}^{k-1} a_{s} f\left(y_{s}\right), \quad v_{n}=c n+\sum_{k=N}^{n-1} \sum_{s=k}^{\infty} b_{s} g\left(x_{s}\right), \quad n \geq N .
$$

The mapping $F$ satisfies the assumptions of Knaster's fixed point theorem [2]: $F$ is increasing and maps into itself. Indeed, if $x \in \Omega$, then

$$
\begin{aligned}
& d n \leq u_{n} \leq d n+n \sum_{s=N}^{\infty} a_{s} f(2 c s) \leq 2 d n, \quad n \geq N, \\
& c n \leq v_{n} \leq c n+n \sum_{s=N}^{\infty} b_{s} g(2 d s) \leq 2 c n, \quad n \geq N .
\end{aligned}
$$

By Knaster's fixed point theorem [2], we can conclude that there exists $(x, y) \in \Omega$ such that $(x, y)=F(x, y)$. That is,

$$
x_{n}=d n+\sum_{k=N}^{n-1} \sum_{s=N}^{k-1} a_{s} f\left(y_{s}\right), \quad y_{n}=c n+\sum_{k=N}^{n-1} \sum_{s=k}^{\infty} b_{s} g\left(x_{s}\right), \quad n \geq N .
$$


Then $\lim _{n \rightarrow \infty} x_{n}=\infty$ and $\lim _{n \rightarrow \infty} y_{n}=\infty$. Hence, $\left\{\left(x_{n}, y_{n}\right)\right\}$ is a positive solution of $(1.1)$ which belongs to $C(\infty, \infty)$. The proof is complete.

\section{The case $A_{n_{0}}<\infty$ and $B_{n_{0}}<\infty$}

We first give a classification scheme for positive solutions of (1.1) under the assumption $A_{n_{0}}<\infty$ and $B_{n_{0}}<\infty$.

Theorem 4.1. Suppose that $A_{n_{0}}<\infty$ and $B_{n_{0}}<\infty$. Then any positive solutions of (1.1) must belong to the following classes:

$$
\begin{aligned}
C(\alpha, \beta) & =\left\{\left(x_{n}, y_{n}\right) \in C \mid \lim _{n \rightarrow \infty} x_{n}=\alpha \geq 0, \lim _{n \rightarrow \infty} y_{n}=\beta>0\right\}, \\
C(\infty, \beta) & =\left\{\left(x_{n}, y_{n}\right) \in C \mid \lim _{n \rightarrow \infty} x_{n}=\infty, \lim _{n \rightarrow \infty} y_{n}=\beta>0\right\}, \\
C(\alpha, \infty) & =\left\{\left(x_{n}, y_{n}\right) \in C \mid \lim _{n \rightarrow \infty} x_{n}=\alpha \geq 0, \lim _{n \rightarrow \infty} y_{n}=\infty\right\}, \\
C(\infty, \infty) & =\left\{\left(x_{n}, y_{n}\right) \in C \mid \lim _{n \rightarrow \infty} x_{n}=\infty, \lim _{n \rightarrow \infty} y_{n}=\infty\right\} .
\end{aligned}
$$

Proof. Let $\left\{\left(x_{n}, y_{n}\right)\right\}$ be a positive solution of (1.1). Then $\Delta^{2} y_{n}=-b_{n} g\left(x_{n}\right)<0$ for $n \geq n_{0}$. Hence $\left\{\Delta y_{n}\right\}$ is monotonic and either $\Delta y_{n}>0$ for $n \geq n_{0}$ or $\Delta y_{n}<0$ for $n \geq n_{0}$. If the later holds, then $y_{n} \leq y_{n_{0}}$ for $n \geq n_{0}$ and $\Delta y_{n} \leq \Delta y_{n_{0}}<0$ for $n \geq n_{0}$, and so $y_{n} \leq y_{n_{0}}+$ $\sum_{n=n_{0}}^{n-1} \Delta y_{n_{0}} \rightarrow-\infty$ as $n \rightarrow \infty$, which contradicts the assumption that $y_{n}>0$ for $n \geq n_{0}$, and means that $\lim _{n \rightarrow \infty} y_{n}=\infty$ or $\lim _{n \rightarrow \infty} y_{n}=\beta>0$. On the other hand, it follows from (1.1) that $\Delta^{2} x_{n}>0$ for $n \geq n_{0}$, which implies that $\left\{\Delta x_{n}\right\}$ is monotonic and either $\Delta x_{n}>0$ for $n \geq n_{0}$ or $\Delta x_{n}<0$ for $n \geq n_{0}$. If the later holds, then $\lim _{n \rightarrow \infty} x_{n}=\alpha \geq 0$. If the former holds, then $\lim _{n \rightarrow \infty} x_{n}=\infty$ or $\lim _{n \rightarrow \infty} x_{n}=\alpha>0$. The proof is complete.

Again, in order to justify our classification schemes, we derive several necessary and/or sufficient conditions for the existence of each type of positive solutions.

Theorem 4.2. Suppose that $A_{n_{0}}<\infty$ and $B_{n_{0}}<\infty$. A necessary and sufficient condition for (1.1) to have a positive solution $\left\{\left(x_{n}, y_{n}\right)\right\}$ which belongs to $C(\alpha, \beta)$ is that

$$
\sum_{k=n_{0}}^{\infty} \sum_{s=k}^{\infty} a_{s} f(c)<\infty, \quad \sum_{s=k}^{\infty} b_{s} g(d)<\infty
$$

for some $c>0$ and $d>0$.

Proof. Let $\left\{\left(x_{n}, y_{n}\right)\right\}$ be a solution of (1.1) such that $\lim _{n \rightarrow \infty} x_{n}=\alpha>0$ and $\lim _{n \rightarrow \infty} y_{n}=$ $\beta>0$. Then there exist four positive constants $c_{1}, c_{2}, c_{3}, c_{4}$ and $N \geq n_{0}$ such that $c_{1} \leq x_{n} \leq$ $c_{2}, c_{3} \leq y_{n} \leq c_{4}$ for $n \geq N$. In view of the first equation of (1.1) and $\lim _{n \rightarrow \infty} x_{n}=\alpha>0$, we have $x_{n}=\alpha+\sum_{k=n}^{\infty} \sum_{s=k}^{\infty} a_{s} f\left(y_{s}\right)$, and so

$$
\sum_{k=n_{0}}^{\infty} \sum_{s=k}^{\infty} a_{s} f\left(c_{3}\right)<\infty
$$


Furthermore, we see from the second equation of (1.1) that $\Delta y_{n}=\sum_{k=n}^{\infty} b_{k} g\left(x_{k}\right)$, and $y_{n}=\beta-\sum_{k=n}^{\infty} \sum_{s=k}^{\infty} b_{s} g\left(x_{s}\right)>0$. Thus,

$$
\sum_{k=n_{0}}^{\infty} \sum_{s=k}^{\infty} b_{s} g\left(c_{1}\right)<\beta<\infty
$$

Conversely, suppose that (4.2) holds. Then there exists $N \geq n_{0}$ such that

$$
\sum_{k=N}^{\infty} \sum_{s=k}^{\infty} a_{s} f(2 c)<d, \quad \sum_{k=N}^{\infty} \sum_{s=k}^{\infty} b_{s} g(2 d)<c
$$

Let $X$ be the Banach space of all real-valued sequences $\left\{\left(x_{n}, y_{n}\right)\right\}$ endowed with the norm

$$
\|(x, y)\|=\max \left\{\sup _{n \geq N}\left|x_{n}\right|, \sup _{n \geq N}\left|y_{n}\right|\right\}
$$

and with the usual pointwise ordering $\leq$. Define a subset $\Omega$ of $X$ as follows:

$$
\Omega=\left\{(x, y) \in X \mid d \leq x_{n} \leq 2 d, c \leq y_{n} \leq 2 c, n \geq N\right\} .
$$

For any subset $B$ of $\Omega$, it is obvious that $\inf B \in \Omega$ and $\sup B \in \Omega$. Let us further define an operator $F: \Omega \rightarrow X$ as follows: for $(x, y) \in X$, let $F(x, y)=(u, v)$ and

$$
u_{n}=d+\sum_{k=n}^{\infty} \sum_{s=k}^{\infty} a_{s} f\left(y_{s}\right), \quad v_{n}=c+\sum_{k=N}^{n-1} \sum_{s=k}^{\infty} b_{s} g\left(x_{s}\right), \quad n \geq N
$$

The mapping $F$ satisfies the assumptions of Knaster's fixed point theorem [2]: $F$ is increasing and maps into itself. Indeed, if $x \in \Omega$, then

$$
\begin{aligned}
& d \leq u_{n}=d+\sum_{k=n}^{\infty} \sum_{s=k}^{\infty} a_{s} f\left(y_{s}\right) \leq d+\sum_{k=n}^{\infty} \sum_{s=k}^{\infty} a_{s} f(2 c) \leq 2 d, \quad n \geq N, \\
& c \leq v_{n}=c+\sum_{k=N}^{n-1} \sum_{s=k}^{\infty} b_{s} g\left(x_{s}\right) \leq c+\sum_{k=N}^{\infty} \sum_{s=k}^{\infty} b_{s} g(2 d) \leq 2 c, \quad n \geq N .
\end{aligned}
$$

By Knaster's fixed point theorem [2], we can conclude that there exists $(x, y) \in \Omega$ such that $(x, y)=F(x, y)$. That is,

$$
x_{n}=d+\sum_{k=n}^{\infty} \sum_{s=k}^{\infty} a_{s} f\left(y_{s}\right), \quad y_{n}=c+\sum_{k=N}^{n-1} \sum_{s=k}^{\infty} b_{s} g\left(x_{s}\right), \quad n \geq N .
$$

Then

$$
\lim _{n \rightarrow \infty} x_{n}=d, \quad \lim _{n \rightarrow \infty} \Delta y_{n}=\lim _{n \rightarrow \infty} \sum_{k=n}^{\infty} b_{k} g\left(x_{k}\right)=0,
$$


and so $\lim _{n \rightarrow \infty} y_{n}=\beta \geq 0$. In view of $\Delta y_{n}=\sum_{k=n}^{\infty} b_{s} g\left(x_{s}\right)>0$, it follows that $\beta>0$. Hence, $\left\{\left(x_{n}, y_{n}\right)\right\}$ is a positive solution of (1.1) which belongs to $C(\alpha, \beta)$. The proof is complete.

By means of similar reasoning used in the proof of Theorems 3.2 and 4.2, we may prove the following three theorems.

Theorem 4.3. Suppose that $A_{n_{0}}<\infty$ and $B_{n_{0}}<\infty$. A sufficient condition for (1.1) to have a positive solution $\left\{\left(x_{n}, y_{n}\right)\right\}$ which belongs to $C(\infty, \beta)$ is that

$$
\sum_{k=n_{0}}^{\infty} \sum_{s=n_{0}}^{k-1} a_{s} f(c)=\infty
$$

for any $c>0$ and

$$
\sum_{k=n_{0}}^{\infty} \sum_{s=k}^{\infty} b_{s} g\left(\sum_{r=n_{0}}^{s-1} \sum_{t=n_{0}}^{r-1} a_{s} f(d)\right)<\infty
$$

for some $d>0$.

Theorem 4.4. Suppose that $A_{n_{0}}<\infty$ and $B_{n_{0}}<\infty$. A sufficient condition for (1.1) to have a positive solution $\left\{\left(x_{n}, y_{n}\right)\right\}$ which belongs to $C(\alpha, \infty)$ is that

$$
\sum_{k=n_{0}}^{\infty} \sum_{s=k}^{\infty} b_{s} g(c)=\infty
$$

for any $c>0$ and

$$
\sum_{k=n_{0}}^{\infty} \sum_{s=k}^{\infty} a_{s} f\left(\sum_{r=n_{0}}^{s-1} \sum_{t=n_{0}}^{r-1} b_{s} g(d)\right)<\infty
$$

for some $d>0$.

Theorem 4.5. Suppose that $A_{n_{0}}<\infty$ and $B_{n_{0}}<\infty$. A sufficient condition for (1.1) to have a positive solution $\left\{\left(x_{n}, y_{n}\right)\right\}$ which belongs to $C(\infty, \infty)$ is that

$$
\sum_{k=n_{0}}^{\infty} a_{k} f(c k)<\infty
$$

for some $c>0$ and

$$
\sum_{k=n_{0}}^{\infty} b_{k} g(d k)<\infty
$$

for some $d>0$. 


\section{The case $A_{n_{0}}<\infty$ and $B_{n_{0}}=\infty$}

In this section, we consider the classification and existence for positive solutions of (1.1) under the assumption $A_{n_{0}}<\infty$ and $B_{n_{0}}=\infty$.

Theorem 5.1. Suppose that $A_{n_{0}}<\infty$ and $B_{n_{0}}=\infty$. Then any positive solutions of (1.1) must belong to the following classes:

$$
\begin{aligned}
C(0, \beta) & =\left\{\left(x_{n}, y_{n}\right) \in C \mid \lim _{n \rightarrow \infty} x_{n}=0, \lim _{n \rightarrow \infty} y_{n}=\beta>0\right\}, \\
C(0, \infty) & =\left\{\left(x_{n}, y_{n}\right) \in C \mid \lim _{n \rightarrow \infty} x_{n}=0, \lim _{n \rightarrow \infty} y_{n}=\infty\right\} .
\end{aligned}
$$

Proof. Let $\left\{\left(x_{n}, y_{n}\right)\right\}$ be a positive solution of (1.1). Then $\Delta^{2} y_{n}=-b_{n} g\left(x_{n}\right)<0$ for $n \geq n_{0}$. Hence $\left\{\Delta y_{n}\right\}$ is monotonic and either $\Delta y_{n}>0$ for $n \geq n_{0}$ or $\Delta y_{n}<0$ for $n \geq n_{0}$. If the later holds, then $y_{n} \leq y_{n_{0}}$ for $n \geq n_{0}$ and $\Delta y_{n} \leq \Delta y_{n_{0}}<0$ for $n \geq n_{0}$, and so $y_{n} \leq y_{n_{0}}+$ $\sum_{n=n_{0}}^{n-1} \Delta y_{n_{0}} \rightarrow-\infty$ as $n \rightarrow \infty$, which contradicts the assumption $y_{n}>0$ for $n \geq n_{0}$, and means that $\lim _{n \rightarrow \infty} y_{n}=\infty$ or $\lim _{n \rightarrow \infty} y_{n}=\beta>0$. On the other hand, it follows from (1.1) that $\Delta^{2} x_{n}>0$ for $n \geq n_{0}$, which implies that $\left\{\Delta x_{n}\right\}$ is monotonic and either $\Delta x_{n}>0$ for $n \geq n_{0}$ or $\Delta x_{n}<0$ for $n \geq n_{0}$. If the former holds, then $x_{n} \geq x_{n_{0}}$ for $n \geq n_{0}$. By the second equation of (1.1) we have

$$
\Delta y_{n}=\Delta y_{n_{0}}-\sum_{k=n_{0}}^{n-1} b_{k} g\left(x_{k}\right) \leq \Delta y_{n_{0}}-g\left(x_{n_{0}}\right) \sum_{k=n_{0}}^{n-1}, \quad b_{k} \longrightarrow-\infty
$$

as $n \rightarrow \infty$, which implies that $\lim _{n \rightarrow \infty} \Delta y_{n}=-\infty$ and hence $\lim _{n \rightarrow \infty} y_{n}=-\infty$, this contradicts the assumption $y_{n}>0$ for $n \geq n_{0}$. If the later holds, then $\lim _{n \rightarrow \infty} x_{n}=\alpha \geq 0$. Since $\Delta x_{n}<0$ for $n \geq n_{0}$, then $x_{n} \geq \alpha \geq 0$ for $n \geq n_{0}$. If $\alpha>0$, then

$$
\Delta y_{n}=\Delta y_{n_{0}}-\sum_{k=n_{0}}^{n-1} b_{k} g\left(x_{k}\right) \leq \Delta y_{n_{0}}-g(\alpha) \sum_{k=n_{0}}^{n-1}, \quad b_{k} \longrightarrow \infty
$$

as $n \rightarrow \infty$, which also contradicts the assumption $y_{n}>0$ for $n \geq n_{0}$. The proof is complete.

In the following, in order to justify our classification schemes, we derive several necessary and/or sufficient conditions for the existence of each type of positive solutions.

Theorem 5.2. Suppose that $A_{n_{0}}<\infty$ and $B_{n_{0}}=\infty$. A necessary and sufficient condition for (1.1) to have a positive solution $\left\{\left(x_{n}, y_{n}\right)\right\}$ which belongs to $C(0, \beta)$ is that

$$
\sum_{k=n_{0}}^{\infty} \sum_{s=k}^{\infty} a_{s} f(c)<\infty
$$

for some $c>0$ and

$$
\sum_{k=n_{0}}^{\infty} \sum_{s=n_{0}}^{k-1} b_{s} g\left(\sum_{r=s}^{\infty} \sum_{t=r}^{\infty} a_{s} f(d)\right)<\infty
$$

for some $d>0$. 
Proof. Let $\left\{\left(x_{n}, y_{n}\right)\right\}$ be a solution of (1.1) such that $\lim _{n \rightarrow \infty} x_{n}=0$ and $\lim _{n \rightarrow \infty} y_{n}=\beta>0$. Then there exist two positive constants $c_{1}, c_{2}$ and $N \geq n_{0}$ such that $c_{1} \leq y_{n} \leq c_{2}$ for $n \geq N$. In view of the first equation of (1.1) and $\lim _{n \rightarrow \infty} x_{n}=0$, we have $\Delta x_{n}=-\sum_{k=n}^{\infty} a_{k} f\left(y_{k}\right)$, and so

$$
\infty>x_{n}=\sum_{k=n}^{\infty} \sum_{s=k}^{\infty} a_{s} f\left(y_{s}\right) \geq \sum_{k=n}^{\infty} \sum_{s=k}^{\infty} a_{s} f\left(c_{1}\right) .
$$

Furthermore, we see from the second equation of (1.1) that $\Delta y_{n}=\sum_{k=n}^{\infty} b_{k} g\left(x_{k}\right)$, and

$$
\begin{aligned}
\infty>y_{n}=y_{N}+\sum_{k=N}^{n-1} \sum_{s=k}^{\infty} b_{s} g\left(x_{s}\right) \geq \sum_{k=N}^{n-1} \sum_{s=k}^{\infty} b_{s} g\left(\sum_{r=s}^{\infty} \sum_{t=r}^{\infty} a_{t} f\left(y_{t}\right)\right) \\
\geq \sum_{k=N}^{n-1} \sum_{s=k}^{\infty} b_{s} g\left(\sum_{r=s}^{\infty} \sum_{t=r}^{\infty} a_{t} f\left(c_{1}\right)\right) .
\end{aligned}
$$

Conversely, suppose that (5.4) and (5.5) hold. Then there exists $N \geq n_{0}$ such that

$$
\sum_{k=N}^{\infty} \sum_{s=k}^{\infty} b_{s} g\left(\sum_{r=s}^{\infty} \sum_{t=r}^{\infty} a_{t} f(2 c)\right) \leq c .
$$

Let $X$ be the Banach space of all real-valued sequences $\left\{y_{n}\right\}$ endowed with the norm $\|y\|=\sup _{n \geq N}\left|y_{n}\right|$ and with the usual pointwise ordering $\leq$. Define a subset $\Omega$ of $X$ as follows:

$$
\Omega=\left\{y \in X \mid c \leq y_{n} \leq 2 c, n \geq N\right\} .
$$

For any subset $B$ of $\Omega$, it is obvious that $\inf B \in \Omega$ and $\sup B \in \Omega$. Let us further define an operator $F: \Omega \rightarrow X$ as follows:

$$
(F y)_{n}=c+\sum_{k=N}^{n-1} \sum_{s=k}^{\infty} b_{s} g\left(\sum_{r=s}^{\infty} \sum_{t=r}^{\infty} a_{t} f\left(y_{t}\right)\right), \quad n \geq N .
$$

The mapping $F$ satisfies the assumptions of Knaster's fixed point theorem [2]: $F$ is increasing and maps into itself. Indeed, if $y \in \Omega$, then

$$
\begin{aligned}
c & \leq(F y)_{n}=c+\sum_{k=N}^{n-1} \sum_{s=k}^{\infty} b_{s} g\left(\sum_{r=s}^{\infty} \sum_{t=r}^{\infty} a_{t} f\left(y_{t}\right)\right) \\
& \leq c+\sum_{k=N}^{\infty} \sum_{s=k}^{\infty} b_{s} g\left(\sum_{r=s}^{\infty} \sum_{t=r}^{\infty} a_{t} f(2 c)\right) \leq 2 c, \quad n \geq N .
\end{aligned}
$$

By Knaster's fixed point theorem [2], we can conclude that there exists a $y \in \Omega$ such that $y=F y$. That is,

$$
y_{n}=c+\sum_{k=N}^{n-1} \sum_{s=k}^{\infty} b_{s} g\left(\sum_{r=s}^{\infty} \sum_{t=r}^{\infty} a_{t} f\left(y_{t}\right)\right), \quad n \geq N .
$$


Set $x_{n}=\sum_{r=n}^{\infty} \sum_{t=r}^{\infty} a_{t} f\left(y_{t}\right)$, then $\lim _{n \rightarrow \infty} x_{n}=0$ and

$$
y_{n}=c+\sum_{k=N}^{n-1} \sum_{s=k}^{\infty} b_{s} g\left(x_{s}\right), \quad n \geq N
$$

and so $\lim _{n \rightarrow \infty} \Delta y_{n}=\lim _{n \rightarrow \infty} \sum_{k=n}^{\infty} b_{k} g\left(x_{k}\right)=0$. Hence $\lim _{n \rightarrow \infty} y_{n}=\beta \geq c>0$ and $\left\{\left(x_{n}\right.\right.$, $\left.\left.y_{n}\right)\right\}$ is a positive solution of $(1.1)$ which belongs to $C(0, \beta)$. The proof is complete.

Theorem 5.3. Suppose that $A_{n_{0}}<\infty$ and $B_{n_{0}}=\infty$. A sufficient condition for (1.1) to have a positive solution $\left\{\left(x_{n}, y_{n}\right)\right\}$ which belongs to $C(0, \infty)$ is that

$$
\sum_{k=n_{0}}^{\infty} b_{k} g\left(\sum_{s=k}^{\infty} \sum_{r=s}^{\infty} a_{r} f(c r)\right)<\infty
$$

for some $c>0$.

Proof. Suppose that (5.14) holds. Then there exists $N$ so large that

$$
\sum_{k=N}^{\infty} b_{k} g\left(\sum_{s=k}^{\infty} \sum_{r=s}^{\infty} a_{r} f(2 c r)\right)<c
$$

Let $X$ be the set of all real-valued sequences $\left\{y_{n}\right\}$ with norm $\sup _{n \geq N}\left|y_{n} / n\right|$. Then $X$ is a Banach space. We define a subset $\Omega$ of $X$ as follows:

$$
\Omega=\left\{y_{n} \in X \mid c n \leq y_{n} \leq 2 c n, n \geq N\right\} .
$$

Then $\Omega$ is a bounded, convex and closed subset of $X$. Let us further define an operator $F: \Omega \rightarrow X$ as follows:

$$
(F y)_{n}=c n+\sum_{k=N}^{n-1} \sum_{s=k}^{\infty} b_{s} g\left(\sum_{r=s}^{\infty}\left(\sum_{t=r}^{\infty} a_{t} f\left(y_{t}\right)\right)\right), \quad n \geq N .
$$

The mapping $F$ satisfies the assumptions of Knaster's fixed point theorem [2]: $F$ is increasing and maps into itself. Indeed, if $x \in \Omega$, then

$$
c n \leq(F y)_{n} \leq c n+\sum_{k=N}^{n-1} \sum_{s=k}^{\infty} b_{s} g\left(\sum_{r=s}^{\infty} \sum_{t=r}^{\infty} a_{t} f(2 c t)\right) \leq 2 c n, \quad n \geq N .
$$

By Knaster's fixed point theorem, we can conclude that there exists a $y \in \Omega$ such that $y=F y$. Set $x_{n}=\sum_{k=n}^{\infty} \sum_{s=k}^{\infty} a_{s} f\left(y_{s}\right)$, then

$$
y_{n}=c n+\sum_{k=N}^{n-1} \sum_{s=k}^{\infty} b_{s} g\left(x_{s}\right)
$$

and $\lim _{n \rightarrow \infty} x_{n}=0$ and $\lim _{n \rightarrow \infty} y_{n}=\infty$. The proof is complete. 


\section{Examples}

In this section, we will give some examples to illustrate our results.

Example 6.1. Consider the following system:

$$
\begin{gathered}
\Delta^{2} x_{n}=a_{n} y_{n}, \\
\Delta^{2} y_{n}=-b_{n} x_{n}, \quad n \geq n_{0},
\end{gathered}
$$

where $a_{n}=6(n+1)$ and $b_{n}=0$. It is easy to see that

$$
\begin{gathered}
A_{n_{0}}=\sum_{n=n_{0}}^{\infty} a_{n}=\sum_{n=n_{0}}^{\infty} 6(n+1)=\infty, \quad B_{n_{0}}=\sum_{n=n_{0}}^{\infty} b_{n}=0<\infty, \\
\sum_{n=n_{0}}^{\infty} \sum_{s=n}^{\infty} b_{s}\left(\sum_{r=n_{0}}^{s-1} \sum_{t=n_{0}}^{r-1} c a_{t}\right)=0<\infty, \quad \text { for some } c>0, \\
\sum_{n=n_{0}}^{\infty} \sum_{s=n_{0}}^{n-1} d a_{s}=\infty, \quad \text { for any } d>0 .
\end{gathered}
$$

By Theorem 3.2, (6.1) has a positive solution $\left\{\left(x_{n}, y_{n}\right)\right\}$ which belongs to $C(\infty, \alpha)$. In fact, it is easy to verify that $x_{n}=n^{3}$ and $y_{n}=1$ is such a positive solution, that is to say, $\Delta^{2} x_{n}=6(n+1)$ and $\Delta^{2} y_{n}=0$.

Example 6.2. Consider the following system:

$$
\begin{gathered}
\Delta^{2} x_{n}=2(\sqrt{n})^{3}\left(\frac{1}{y_{n}}\right)^{3}, \\
\Delta^{2} y_{n}=-\frac{\sqrt{n+2}-\sqrt{n}}{(\sqrt{n+2}+\sqrt{n+1})(\sqrt{n+1}+\sqrt{n}) n^{2}} x_{n}, \quad n \geq n_{0} .
\end{gathered}
$$

Obviously,

$$
\begin{gathered}
A_{n_{0}}=\sum_{n=n_{0}}^{\infty} a_{n}=\sum_{n=n_{0}}^{\infty} 2(\sqrt{n})^{3}=\infty, \\
B_{n_{0}}=\sum_{n=n_{0}}^{\infty} b_{n}=\sum_{n=n_{0}}^{\infty} \frac{\sqrt{n+2}-\sqrt{n}}{(\sqrt{n+2}+\sqrt{n+1})(\sqrt{n+1}+\sqrt{n}) n^{2}}<\infty .
\end{gathered}
$$

Furthermore,

$$
\begin{gathered}
\sum_{n=n_{0}}^{\infty} a_{n} f(c n)=\frac{2}{c^{3}} \sum_{n=n_{0}}^{\infty}(\sqrt{n})^{3} \frac{1}{n^{3}}=\frac{2}{c^{3}} \sum_{n=n_{0}}^{\infty} n^{-3 / 2}<\infty, \quad \text { for some } c>0, \\
\sum_{n=n_{0}}^{\infty} b_{n} g(c n)=c \sum_{n=n_{0}}^{\infty} \frac{\sqrt{n+2}-\sqrt{n}}{(\sqrt{n+2}+\sqrt{n+1})(\sqrt{n+1}+\sqrt{n}) n}<\infty, \quad \text { for some } c>0 .
\end{gathered}
$$


By Theorem 3.3, (6.3) has a positive solution which belongs to $C(\infty, \infty)$. In fact, $x_{n}=n^{2}$ and $y_{n}=\sqrt{n}$ is such a positive solution.

Similarly, we can provide some examples to illustrate our results in Sections 4, 5, and 6.

As a final remark, our results can be extended without too much difficulty to the following two-dimensional delay difference system:

$$
\begin{gathered}
\Delta^{2} x_{n}=a_{n} f\left(y_{n-\tau}\right), \\
\Delta^{2} y_{n}=-b_{n} g\left(x_{n-\delta}\right),
\end{gathered}
$$

where $\tau$ and $\delta$ are positive integers.

\section{Acknowledgments}

The first author was supported by the NNSF of China (10571078) and the Teaching and Research Award Program for Outstanding Young Teachers in Higher Education.

\section{References}

[1] R. P. Agarwal, Difference Equations and Inequalities, Academic Press, New York, 2002.

[2] R. P. Agarwal, M. Bohner, and W.-T. Li, Nonoscillation and Oscillation Theory for Functional Differential Equations, Monographs and Textbooks in Pure and Applied Mathematics, vol. 267, Marcel Dekker, New York, 2004.

[3] S. S. Cheng, On a class of fourth-order linear recurrence equations, International Journal of Mathematics and Mathematical Sciences 7 (1984), no. 1, 131-149.

[4] S. S. Cheng and W. T. Patula, An existence theorem for a nonlinear difference equation, Nonlinear Analysis. Theory, Methods \& Applications 20 (1993), no. 3, 193-203.

[5] X. Z. He, Oscillatory and asymptotic behavior of second order nonlinear difference equations, Journal of Mathematical Analysis and Applications 175 (1993), no. 2, 482-498.

[6] J. W. Hooker and W. T. Patula, Growth and oscillation properties of solutions of a fourth order linear difference equation, Journal of the Australian Mathematical Society, Series B 26 (1985), no. 3, 310-328.

[7] H.-F. Huo and W.-T. Li, Oscillation of the Emden-Fowler difference systems, Journal of Mathematical Analysis and Applications 256 (2001), no. 2, 478-485.

[8] W.-T. Li, Classification schemes for nonoscillatory solutions of two-dimensional nonlinear difference systems, Computers \& Mathematics with Applications 42 (2001), no. 3-5, 341-355.

[9] W.-T. Li and R. P. Agarwal, Positive solutions of higher-order nonlinear delay difference equations, Computers \& Mathematics with Applications 45 (2003), no. 6-9, 1203-1211.

[10] W.-T. Li and S. S. Cheng, Classifications and existence of positive solutions of second order nonlinear neutral difference equations, Funkcialaj Ekvacioj 40 (1997), no. 3, 371-393.

[11] Characterizing conditions for the existence of nonoscillatory solutions of a discrete EmdenFowler equation with summable coefficients, Dynamic Systems and Applications 9 (2000), no. 3, 457-461.

[12] W.-T. Li, S. S. Cheng, and G. Zhang, A classification scheme for nonoscillatory solutions of a higher order neutral nonlinear difference equation, Journal of the Australian Mathematical Society, Series A 67 (1999), no. 1, 122-142.

[13] W.-T. Li and Y. N. Raffoul, Classification and existence of positive solutions of systems of Volterra nonlinear difference equations, Applied Mathematics and Computation 155 (2004), no. 2, 469478. 
[14] J. Popenda and E. Schmeidel, On the solutions of fourth order difference equations, The Rocky Mountain Journal of Mathematics 25 (1995), no. 4, 1485-1499.

[15] B. Smith and W. E. Taylor Jr., Oscillatory and asymptotic behavior of certain fourth order difference equations, The Rocky Mountain Journal of Mathematics 16 (1986), no. 2, 403-406.

[16] W. E. Taylor Jr., Oscillation properties of fourth order difference equations, Portugaliae Mathematica 45 (1988), no. 1, 105-114.

[17] E. Thandapani and I. M. Arockiasamy, Fourth-order nonlinear oscillations of difference equations, Computers \& Mathematics with Applications 42 (2001), no. 3-5, 357-368.

[18] E. Thandapani, R. Arul, J. Graef, and P. W. Spikes, Asymptotic behavior of solutions of second order difference equations with summable coefficients, Bulletin of the Institute of Mathematics. Academia Sinica 23 (1995), 343-356.

[19] J. Yan and B. Liu, Oscillatory and asymptotic behaviour of fourth order nonlinear difference equations, Acta Mathematica Sinica. New Series 13 (1997), no. 1, 105-115.

[20] B. G. Zhang and S. S. Cheng, On a class of nonlinear difference equations, Journal of Difference Equations and Applications 1 (1995), no. 4, 391-411.

Wan-Tong Li: School of Mathematics and Statistics, Lanzhou University, Lanzhou, Gansu 730000, China

E-mail address: wtli@lzu.edu.cn

Can-Yun Huang: School of Mathematics and Statistics, Lanzhou University, Lanzhou, Gansu 730000, China

E-mail address: hcy@lut.cn

Sui Sun Cheng: Department of Mathematics, National Tsing Hua University, Hsinchu, Taiwan 30043, China

E-mail address: sscheng@math.nthu.edu.tw 


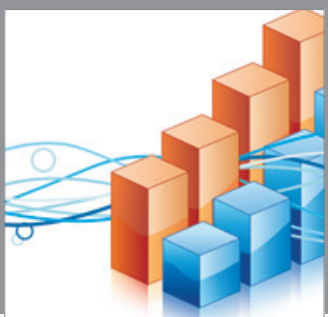

Advances in

Operations Research

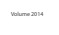

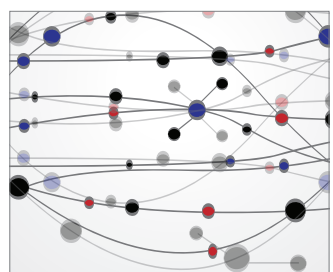

\section{The Scientific} World Journal
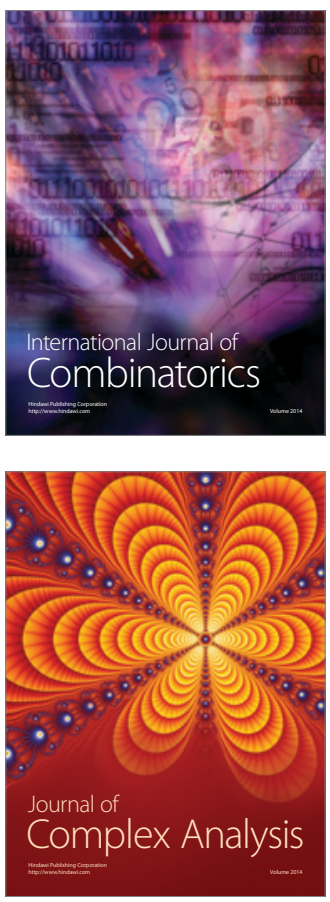

International Journal of

Mathematics and

Mathematical

Sciences
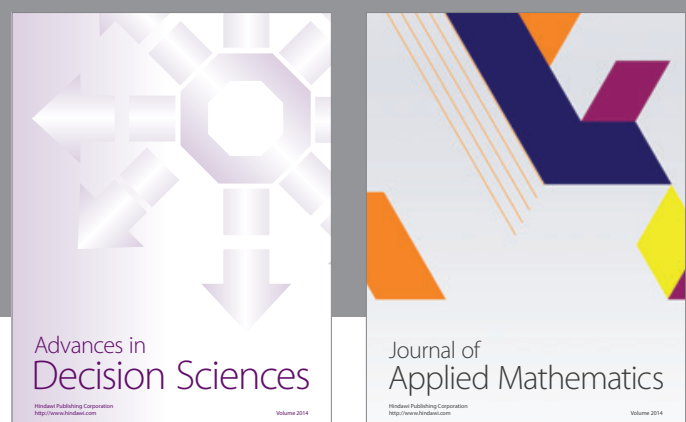

Journal of

Applied Mathematics
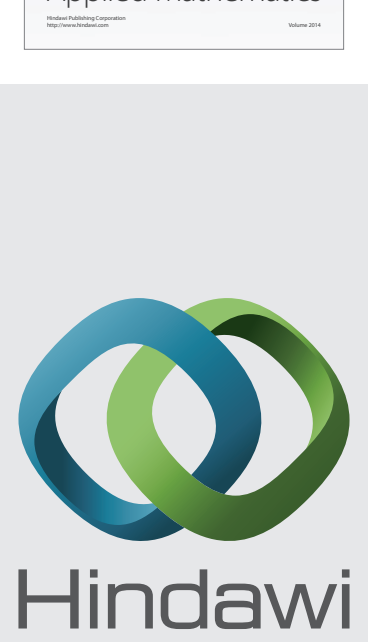

Submit your manuscripts at http://www.hindawi.com
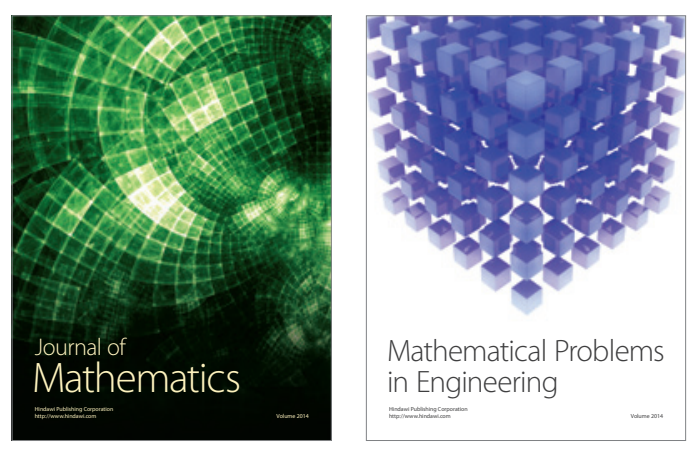

Mathematical Problems in Engineering
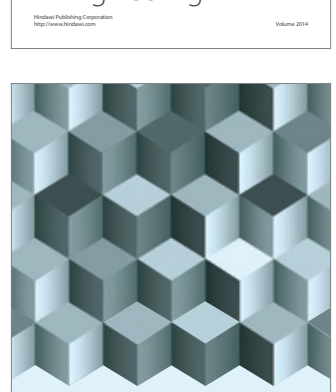

Journal of

Function Spaces
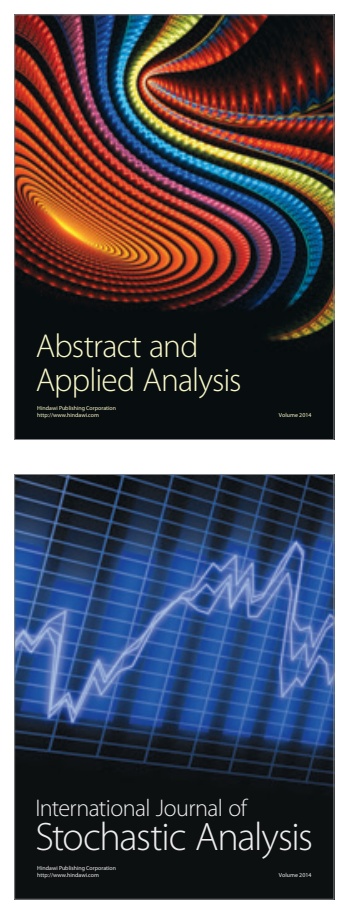

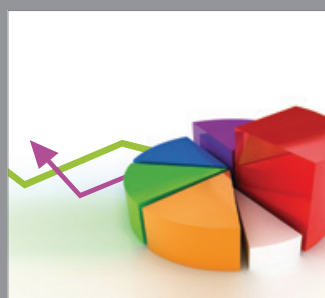

ournal of

Probability and Statistics

Promensencen
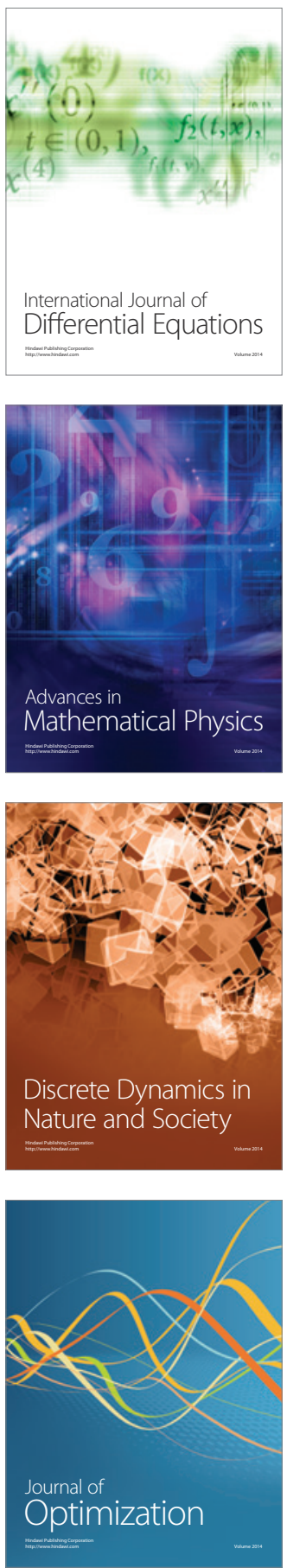